\title{
Exclusión contra homosexuales y lesbianas en el Estado de Hidalgo, México. Aproximaciones al perfil de sujeto discriminante
}

Discrimination against homosexuals and lesbians in the state of Hidalgo, Mexico. Approaches to the profiling of agents of discrimination

\section{Carlos Mejía Reyes}

Instituto de Ciencias Sociales y Humanidades, Universidad Autónoma del Estado de Hidalgo, México

Red Internacional América Latina, Europa y el Caribe (Red-ALEC). Limoges, Francia carlos_mejia7563@uaeh.edu.mx

\section{Edgar Noé Blancas Martínez}

Instituto de Ciencias Sociales y Humanidades, Universidad Autónoma del Estado de Hidalgo, México

noeb@uaeh.edu.mx

\section{Abraham Sánchez Ruiz \\ Universidad De La Salle Bajío, Campus Salamanca, México \\ asanchezr@delasalle.edu.mx}

Recibido: 01/04/2018 • Aceptado: 05/03/2019

\section{RESUMEN}

La discriminación contra homosexuales y lesbianas en México se percibe como una problemática estructural y ampliamente extendida. Tras dos encuestas nacionales que estudian su tendencia, se ha detectado a este sector poblacional como de los más violentados, al grado de que se ubica como el segundo país con mayores índices de crímenes por homofobia. Sin embargo, poco se ha estudiado este fenómeno en las entidades que conforman la república. Así, este documento hace un 
acercamiento al fenómeno de exclusión en el Estado de Hidalgo, México, a partir de la Encuesta Nacional sobre Discriminación de 2010. El procedimiento metodológico se conforma por una revisión estadística descriptiva para examinar la tendencia y se construyen perfiles analíticos de agentes discriminadores mediante la técnica de correspondencias múltiples con variables demográficas básicas.

Palabras clave: Discriminación; homosexuales; lesbianas; Estado de Hidalgo, México.

\section{ABSTRACT}

Discrimination against homosexuals and lesbians in Mexico is perceived as a structural and widespread problem. Two national surveys that study its characteristics identify this population segment as one of the most common targets of violence, with the second-highest rates of homophobic crimes in the world. However, governments have made little effort to study this phenomenon. This document therefore investigates the phenomenon of exclusion in the State of Hidalgo, Mexico, using data from the 2010 National Survey on Discrimination. The methodological approach consists of a descriptive statistical review to examine trends and the construction of analytical profiles of agents of discrimination based on the technique of multiple correspondences with basic demographic variables.

Keywords: Discrimination, homosexuals, lesbians, state of Hidalgo, Mexico.

\section{INTRODUCCIÓN}

La Carta de las Naciones Unidas de 1945 y la Declaración Universal de Derechos Humanos de 1948 sentaron las bases jurídicas de protección a la igualdad y equidad en sujetos de orientaciones, identidades y preferencias sexuales diversas. La ratificación por parte de los Estados constituyentes del pacto se comprometió explícitamente a la vigilancia de su cumplimiento mediante la incorporación de sus máximas en las cartas rectoras de organización social y jurídica.

Paradójicamente, el prejuicio contra las personas no heterosexuales se mantuvo arraigado en todos los niveles de la vida cotidiana (política, 
científica y social) como consecuencia del traslado de las máximas religiosas de herencia judeo-cristiana a los parámetros legítimos del saber cotidiano (Cabral, 2017). Un ejemplo de ello fue señalado por el sociólogo Erving Goffman (2006) en 1964, al referir a la homosexualidad como uno de los estigmas detectados más comunes en su contexto y clasificado como "pasiones antinaturales". Esto a pesar de Alfred Kinsey, Pomeroy y Martin (2003), demostraran en 1948 que las prácticas sexuales no se someten al esquema binario típico, ya que el $37 \%$ de los varones heterosexuales entrevistados refirieron alguna experiencia homosexual entre el inicio de la adolescencia y la vejez.

Igualmente en 1949, el psicoanalista Medrad Boss divulgó la terapia de una mujer "homosexual neurótica" y otra denominada "constitucional", deduciendo como razones de sus identidades a trastornos infantiles no superados equivalentes a hombres amputados de los genitales (Mogrovejo, 2000). Incluso, el prejuicio se trasladó a las explicaciones científicas en manuales de salud pública, desde los cuales las instancias gubernamentales emitieron diagnósticos. Sin embargo, fue hasta 1970 que la homosexualidad dejó de ser considerada una patología mental en el mundo occidental (Barrientos, 2016).

En este contexto, 1972, el término "homofobia" surge en el ámbito académico por el libro de George Weimberg Society and the Healthy Homosexual; permitiendo sintetizar en un concepto el tipo de exclusión, objeto de temor y violencia que padecen las personas no heterosexuales (Tena, 2012). Aunque décadas después se demostró que es el odio o disgusto irracional -en lugar del miedo- lo que orienta el desprecio hacia este sector poblacional (Herek, 2004). A pesar de todo lo anterior, en la década de los años 80 , se responsabilizó directamente a los homosexuales de ser portadores inherentes del VIH-SIDA, al grado de prohibirles abiertamente la donación de sangre (Tena, 2012).

En el contexto mexicano de la década de los años 60, la homosexualidad era considerada un delito cuyo castigo fue la cárcel sin garantías legales, sin defensores ni proceso. Al mismo tiempo, la lesbiandad padecía categórica invisibilidad (Rivera, Tirado, y Olea, 2014), incluso en eventos internacionales destinados a la atención de los derechos de las mujeres en décadas siguientes (Valencia y Romero, 2017). Más recientemente, en la década de los 90 , los contenidos escolares en educación básica de la zona 
occidental del país señalaban a la homosexualidad como "una costumbre desviada" (Monsiváis, 2012).

Fue hasta la reforma de 2001 de la Constitución Política de los Estados Unidos Mexicanos que se incorporó la innovación que prohíbe la discriminación por preferencias sexuales, cuyo primer artículo indica:

Queda prohibida toda discriminación motivada por origen étnico o nacional, el género, la edad, las capacidades diferentes, la condición social, las condiciones de salud, la religión, las opiniones, las preferencias, el estado civil o cualquier otra que atente contra la dignidad humana y tenga por objeto anular o menoscabar los derechos y libertades de las personas (Diario Oficial de la Federación, 14 de agosto de 2001).

De este ordenamiento es que en 2003 se creó la Ley Federal para Prevenir y Erradicar la Discriminación (LFPED), que establece en el artículo dieciséis la definición del término como:

[...] toda distinción, exclusión, restricción o preferencia que, por acción u omisión, con intención o sin ella, no sea objetiva, racional ni proporcional y tenga por objeto o resultado obstaculizar, restringir, impedir, menoscabar o anular el reconocimiento, goce o ejercicio de los derechos humanos y libertades, cuando se base en uno o más de los siguientes motivos: el origen étnico o nacional, el color de piel, la cultura, el sexo, el género, la edad, las discapacidades, la condición social, económica, de salud o jurídica, la religión, la apariencia física, las características genéticas, la situación migratoria, el embarazo, la lengua, las opiniones, las preferencias sexuales, la identidad o la filiación política, el estado civil, la situación familiar, las responsabilidades familiares, el idioma, los antecedentes penales o cualquier otro motivo; también se entenderá como discriminación la homofobia, la misoginia, cualquier manifestación de xenofobia, segregación racial, antisemitismo, así como la discriminación racial y otras formas conexas de intolerancia (LFPED, 2003, art. 16).

En la posterior reforma constitucional de 2011, establece la máxima carta legal del país en el artículo primero: 
Queda prohibida toda discriminación motivada por origen étnico o nacional, el género, la edad, las discapacidades, la condición social, las condiciones de salud, la religión, las opiniones, las preferencias sexuales, el estado civil o cualquier otra que atente contra la dignidad humana y tenga por objeto anular o menoscabar los derechos y libertades de las personas (Constitución Política de los Estados Unidos Mexicanos, 2016, art. 1).

A pesar de los ordenamientos, México ha sido caracterizado como el segundo país con mayores indicadores de crímenes por homofobia en 2015 (Pantoja, 2015), ya que desde 1995 se han registrado mil trecientos diez asesinatos en veintinueve de treinta y dos entidades federativas. Lo que significa que se comenten sesenta y cinco homicidios al año; cinco al mes; uno por semana (Becerro-Acosta, 2016).

Los datos anteriores constituyen un indicador de la persistencia en las actitudes contra sujetos considerados susceptibles de exclusión social por su identidad u orientación sexual. Tan es así, que en la primera Encuesta Nacional sobre Discriminación de 2005 , el $48.8 \%$ de la muestra nacional refirió que no permitiría que en su casa viviera una persona homosexual; siendo este el sector poblacional con mayores porcentajes de señalamiento negativo por encima de extranjeros $(42.1 \%)$, personas con ideas políticas distintas $(38.3 \%)$, de otra religión $(36.2 \%)$, de otra raza $(31.8 \%)$, indígenas $(20.1 \%)$ y con discapacidad (15\%). Lo cual se refleja en el $94.7 \%$ de personas homosexuales que sintieron actitudes discriminatorias en su contra (Consejo Nacional Para Prevenir la Discriminación y Secretaría de Desarrollo Social, 2005).

Para la encuesta de 2010 , es el $44 \%$ de la muestra el que refirió que no permitiría a una persona homosexual vivir en su hogar, mientras que el $43.5 \%$ lo puntualizó con lesbianas (Consejo Nacional para Prevenir la Discriminación, 2010). Al igual que el levantamiento anterior, este sector fue el más discriminado, ya que solamente el $35.9 \%$ permitiría tal proximidad con personas de otra religión, el $25.5 \%$ con personas de ideas políticas distintas a las suyas, $25.8 \%$ con extranjeros, $25 \%$ con $\mathrm{VIH}, 24.9 \%$ con personas que llegan de fuera, de otra raza $22.8 \%$, $22.7 \%$ con personas de culturas distintas y $13.6 \%$ con personas con discapacidad. De tal forma que el señalamiento negativo en México es constante contra personas de la diversidad sexual. 
Diversos estudios empíricos previos han documentado la relación entre condiciones personales con el prejuicio negativo contra personas no heterosexuales. Por ejemplo, la edad es utilizada en su influencia discriminatoria (Barrientos y Cárdenas, 2010; Coppari, et al., 2014; Acuña-Ruiz y Oyuela, 2006), así como en actitudes de autoreprobación (Herek, Cogan, Gillis, y Glunt, 1998) o autoestigma (Herek, Gillis, y Cogan, 2015) cuando identifican su orientación, preferencia o identidad sexual. La relación de la edad con actitudes discriminatorias ha sido demostrado que asevera la propensión de informantes de menor edad con niveles bajos de rechazo hacia personas no heterosexuales (Piña y Aguayo, 2015; Steffensy\& Wagner, 2004). Por lo tanto, se usará la variable (p8), recodificada en diez rangos, atendiendo a los parámetros que los organismos públicos mexicanos establecen para clasificar a sujetos con minoría de edad (hasta 17 años), jóvenes (18 a 25, 26 a 30), adultos ( 31 a 35, 36 a 40, 41 a 45, 46 a 50, 51 a 55,56 a 60$)$ y adultos mayores (60 años o más).

El sexo ha sido recurrentemente utilizado para realizar predicciones estadísticas de informantes con prejuicios homofóbicos o lesbofóbicos (Gabriel y Banse, 2006; Jarayatne, et al., 2006). Se ha encontrado que son los hombres menos tolerantes y con más distancia o prejuicio contra homosexuales en comparación con las mujeres (Fernández y Calderón, 2014; Coppari, et al., 2014) e incluso contra las lesbianas (Lozano y Rocha, 2011; LaMar y Kite, 1998). En cuanto a las actitudes de aprobación hacia el matrimonio de personas del mismo sexo, de igual forma los varones reportaron mayores índices de desautorización, a diferencia de las mujeres (Smith-Castro y Molina-Delgado, 2011).

Otra es la formación escolar. Se ha demostrado que es un predictor básico e, incluso, un factor determinante en la superación de prejuicios homofóbicos (Jones y Sullivan, 2008). Diversos estudios teóricos (Herek, 1984; Herek, 2000) así como empíricos han demostrado la relación entre altos niveles de educación con los bajos índices de prejuicio en general (Pratto, Sidanius, Stallworth, y Malle, 1994) así como hacia lesbianas y homosexuales (Herek y Capitanio, 1996; Morrison y Morrison, 2011). La relación empírica hallada en las disertaciones previas sobre el tema de la clase social refiere que entre más alta la clase social, menos intolerancia o actitudes reprobatorias hacia homosexuales y lesbianas (Pratto et al., 1994; Herek y Capitanio, 1996; Morrison y Morrison, 2011; Swank, Fash y Frost, 2013). 
La pertenencia o filiación religiosa ha sido históricamente una variable que predice la tendencia actitudinal negativa hacia identidades y preferencias no heterosexuales. Desde los orígenes de la organización cultural de las sociedades occidentalizadas, se ha documentado que la organización social de la sexualidad respondió a las necesidades de los colectivos, calificando a la homosexualidad y lesbiandad como reprobable para el intercambio de mujeres entre hordas (Rubin, 2003). Dicho mandato se estableció en campos simbólicos e imaginarios (Serret, 2004) que, institucionalizados a partir de mitos, se implantaron como mandatos religiosos (Serret, 2001; Cabral, 2017). Así, la religión sacraliza lo simbolizado colectivamente (Durkheim, 2000) y lo traduce en ordenanzas, que en este caso, prohíben y castigan la no heterosexualidad (Alexandre, 2001). A nivel de estudios empíricos, la pertenencia o filiación religiosa influyen en la reprobación hacia matrimonios entre personas del mismo sexo (Smith-Castro y Molina-Delgado, 2011), así como en contra de contratar personas no heterosexuales en empleos (Berg y Lien, 2002) y de manera general entre mayor religiosidad, mayor prejuicio (Piña y Aguayo, 2015; Toro y Varas, 2004; Horvarth y Ryan, 2003; Laythe, Finkel y Kirkpatrick, 2001).

Con estos antecedentes, el estudio tiene por objetivo aproximarse al fenómeno de la discriminación en el Estado de Hidalgo, México, de dos maneras: en primer lugar, comparar los resultados de la Encuesta Nacional de Discriminación con la submuestra hidalguense con respecto a actitudes excluyentes contra homosexuales y lesbianas. En segundo lugar, construir un perfil analítico de informante discriminador contra este sector, cuya hipótesis es que se conforma por informantes de edades adultas, poco formadas académicamente, de clase social baja, orientación religiosa judeo-cristiana y, particularmente, varón.

Previo a proceder, es menester describir al Estado de Hidalgo, México, para ubicar contextualmente el espacio donde se centra el estudio. Hidalgo tiene una superficie de $20813 \mathrm{~km}^{2}$, que representan el $1.1 \%$ del territorio nacional. Posee 64 municipios, de los cuales Pachuca de Soto es el centro político administrativo. Su población, en el año 2010, fue de 2 665018 de habitantes, de los que $51.7 \%$ son varones y el resto, $48.3 \%$, mujeres; en conjunto, representan el $2.4 \%$ de la población nacional. La distribución poblacional en $52 \%$ se ubica en localidades urbanas y el resto en parajes rurales (Franco, 2012). 
La mitad de la población de la entidad se conforma por personas de edades entre 15 y 30 años, con esperanza de vida promedio de 73.6 años (Fernández, 2014). El promedio de formación escolar es de ocho años de estudios y el porcentaje de analfabetismo es de poco más del $6 \%$. También, en este estado se ubican tres grupos indígenas: Nahuas, Otomíes y Tepehuas. Se emplean nueve lenguas: nahua, otomí, tepehua, mixteco, zapoteco, totonaca, mazahua, maya y huasteco; lo que equivale al $15 \%$ de la población que habla alguna lengua indígena.

Las principales actividades económicas son el comercio, construcción, transporte e industria alimentaria, que contribuyen al PIB nacional en $1.5 \%$. Sin embargo, es importante señalar que el estado se ubica entre las primeras entidades con mayor marginación del país, así como uno de los principales estados expulsores de migrantes hacia Estados Unidos de América (Franco, 2012).

Con respecto al tema de discriminación en la entidad, los estudios académicos son escasos. Solamente la Asociación Civil llamada Academia Hidalguense de Educación y Derechos Humanos ha tenido investigaciones sistemáticas sobre el tema y han sido expuestas en obras puntuales que tangencialmente aportan conocimiento al respecto, pero que resultan fuentes imprescindibles de consulta para profundizar. La obra de Vargas (2007) como inaugural y los estudios de opinión de Castelán $(2016 ; 2017)$ elaboró en la ciudad capital, específicamente orientado al del presente artículo, son los únicos. De modo que este estudio pretende contribuir al estado de conocimiento sobre el tema en la entidad con base en datos oficiales para describir aproximativamente el fenómeno.

\section{METODOLOGÍA}

Esta investigación utiliza la Encuesta Nacional sobre Discriminación (ENADIS) realizada en 2010. La peculiaridad fundamental que legitima su uso es que hasta el primer semestre de 2018 conforma la única fuente de datos existente y oficial, de carácter nacional, que permite el análisis cuantitativo de las actitudes discriminatorias hacia diversos sectores poblacionales identificados como típicamente vulnerables (mujeres, niñas y niños, jóvenes, adultos mayores, migrantes, "minorías" religiosas, étnicas, culturales y sexuales, personas trabajadoras del hogar y con alguna "capacidad diferente” C Consejo Nacional para Prevenir la Discriminación, 2011, p. 9). 


\section{Participantes}

La ENADIS fue elaborada utilizando un cuestionario general aplicado a hogares y otro de manera específica hacia a un integrante familiar aleatoriamente elegido para captar los valores y actitudes referentes a la discriminación. Así, la muestra consistió en un número de casos $(N)$ de 13751 hogares y 52095 informantes de treinta y dos entidades federativas que componen la república mexicana y en 31 municipios (Barba, 2012).

Para la submuestra del Estado de Hidalgo, se utilizaron los resultados de 688 informantes. De los cuales, $51.5 \%$ son mujeres y el restante $48.5 \%$ son varones. La media de edad es de 32 años. El porcentaje de informantes sin instrucción educativa es de $4.8 \%$; el $65.4 \%$ tiene educación básica; $20.7 \%$ tiene educación media y el $8.5 \%$ con educación profesional. El $55.1 \%$ es empleado, mientras que el resto, $44.9 \%$, no labora.

\section{Instrumentos}

Las variables dependientes a utilizar para el análisis versan de la siguiente manera:

012_3.- ¿Estaría dispuesto o no estaría dispuesto a permitir que en su casa vivieran personas homosexuales?

012_9.- ¿Estaría dispuesto o no estaría dispuesto a permitir que en su casa vivieran personas lesbianas?

Las respuestas de ambas interrogantes son: 1) Sí. 2) Sí, en parte. 3) No.

El porcentaje de respuesta a esta pregunta en la submuestra fue de cien por ciento, por lo que su nivel de validez y factibilidad es adecuada. Además, se consideró la dimensión última como la nodal para el análisis, ya que representa la intensidad actitudinal negativa contra homosexuales y lesbianas que resulta es útil para atender los objetivos planteados.

Como variables independientes, se consideró el sexo (p7), variable dicotómica de control. La escolaridad (P12n) está compuesta por once dimensiones que parten de informantes sin formación escolar, preescolar, primaria, secundaria, carrera técnica con secundaria, preparatoria o bachillerato, 
carrera técnica con preparatoria, estudios normalistas, profesional, maestría o doctorado y carrera secretarial con primaria. La variable religión (P37) tiene doce categorías: católico, cristiano, evangélico, bautista, mormón, testigo de jehová, islam, budista, hindú, sin religión y otras. La edad se reconfiguró de una variable continua a una de intervalo; el criterio fue que en la primera categoría se congreguen la minoría de edad legal ( 1 a 17 años), la segunda de 18 a 25 años y, posteriormente, en intervalos de cinco; quedando así doce dimensiones de la variable.

Por último, la clase social como factor independiente fue construida a partir de recategorizar una variable que registró ingresos brutos para convertirla en intervalos conforme los marcos referenciales que las instituciones mexicanas establecieron para definir estratos económicos. Según la Secretaría de Economía, la clase social denominada pobreza extrema se ubica en el rango de ingresos mensuales de 0.0 a 2699 pesos mexicanos; la clase pobre, de 2700 a 6799 pesos; clase media baja de 6800 a 11 599; clase media de 11600 a 34 999; clase media alta de 35000 a 84 999; y la clase alta con ingresos mayores a 85000 (Aguayo, 2014; González, 2015).

\section{Procedimientos}

El modo inicial de análisis es de corte comparativo. Esta técnica descriptiva tiene por objetivo, y proceso, el examen de semejanzas y diferencias para extraer primeras conjeturas (Colino, 2009), así como relaciones entre variables, pero no únicamente como método de medición (Lijphart, 1971). Es útil, especialmente, para dar cuenta de especificidades del fenómeno a estudiar (Mills, Van de Bunt, y De Brujin, 2006) y posteriormente proponer regularidades de fenómenos sociales que permitan generar modelos explicativos o testar hipótesis (Vigour, 2011).

Con lo anterior, el procedimiento compara la dimensión negativa (de actitud discriminatoria) de la variable dependiente en la submuestra objeto de estudio con el resto de submuestras correspondientes a las otras entidades federativas, así como con el promedio nacional, para identificar posiciones porcentuales. Esto es factible porque, de acuerdo con los lineamientos mínimos de la metodología comparativa, se corresponden en observar a un mismo problema de investigación o hechos a indagar, es homogéneo en la recolección de datos y sin diferencias a nivel de poblaciones encuestadas (Colino, 2009). 
Enseguida, se empleará la técnica de análisis de correspondencias múltiples, que es un procedimiento multivariante típico para esta pauta de estudios, porque las variables analizadas son de corte cualitativas/ordinales. Esto ayuda a simplificar su relación a partir de un gráfico en dos longitudes (Visauta y Martori, 2003; Greenacre y Hastie, 1987). El objetivo de esta técnica es determinar la posición de los casos y dimensiones de las variables a partir de sus propiedades en un espacio vectorial de dos ejes y, con ello, señalar relación o proximidad (Sourial et al., 2010; Visauta y Martori, 2003). Con esta distribución espacial se pueden deducir tipologías de informante (López, 1996; Lozares, 1990), pero más precisamente perfiles analíticos (Inglehart y Welzel, 2006). La ventaja de este procedimiento radica en que prescinde de las medidas de asociación entre variables como la chi cuadrada o coeficientes de correlación, ya que proporciona una medida de agrupación más precisa que otras técnicas multivariantes (Inercia y Alfa de Cronbach) (Sourial, et al., 2010).

\section{RESULTADOS}

Los resultados del análisis estadístico descriptivo inicial permiten ubicar el nivel o porcentaje nacional que la muestra refirió con respecto a las actitudes discriminatorias contra homosexuales y lesbianas. En primer, lugar se observa el rasgo nacional sobre el tema, en donde el $44 \%$ de los y las informantes refirieron que no permitirían que una persona homosexual viviera en su hogar (Figura 1). Con este primer dato, el análisis compara el resto de las submuestras del país, ubicando el caso puntual de Hidalgo.

La entidad federativa que señala mayores porcentajes de informantes con intolerancia es Oaxaca, con $56.6 \%$, y el que en menor medida lo señaló fue en el caso de Sinaloa con $31 \%$. Hidalgo se ubicó con $47.5 \%$; es decir, $16.5 \%$ puntos porcentuales por encima de la mínima y $3.5 \%$ de la media, ubicándose en el conjunto de submuestras más intolerantes. Por lo tanto, se posiciona en el lugar 12 de 32 estados con mayores índices de sectarismo contra varones no heterosexuales.

Para el caso de las actitudes con respecto a lesbianas, los resultados no difieren demasiado en cuanto porcentajes y posición comparativa con la media nacional al resto de submuestras (Figura 2). 
Estaría dispuesto o no estaría dispuesto a permitir que en su casa vivieran personas...? Homosexuales

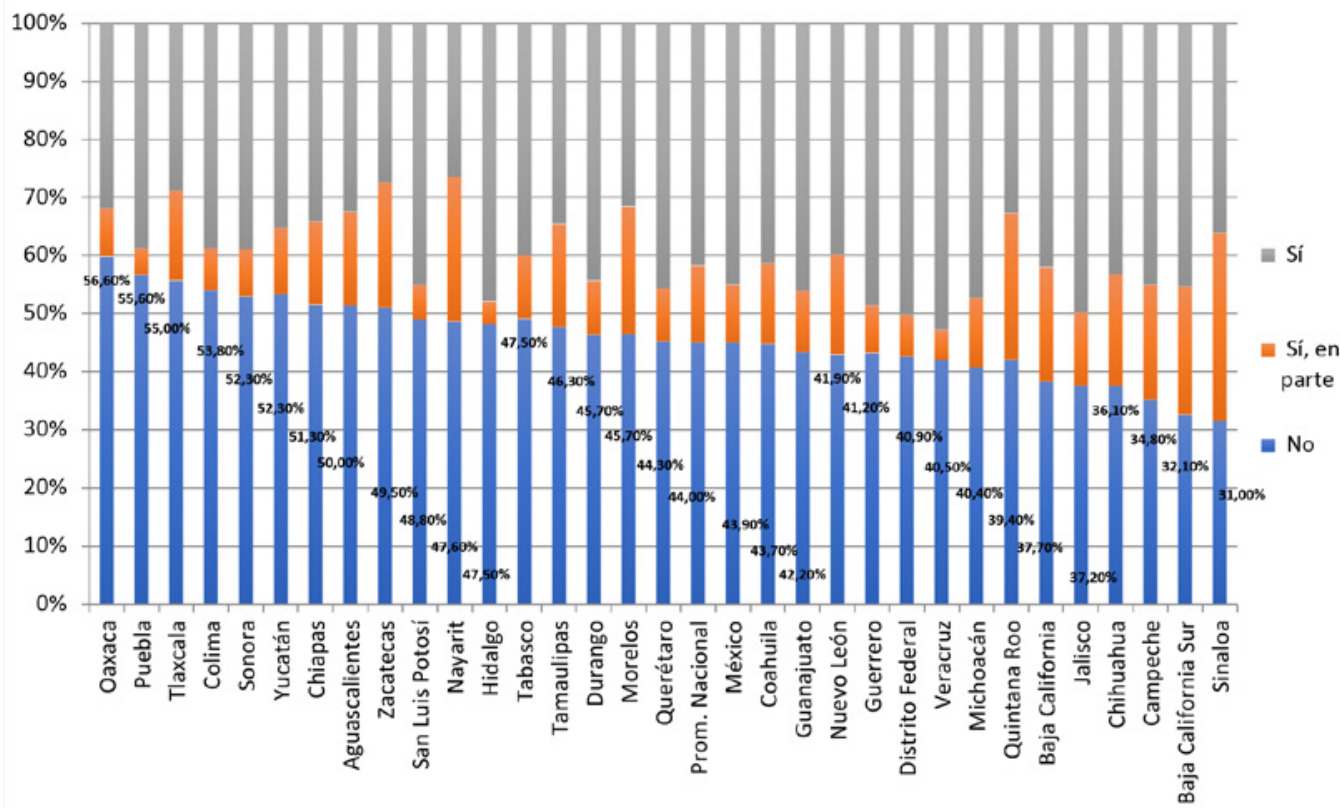

Figura 1. Actitudes discriminatorias contra homosexuales. Comparativo porcentual México y entidades federativas. Fuente: Elaboración propia con base en ENADIS 2010.

El $43.5 \%$ de informantes de la muestra nacional reprueba a lesbianas como personas admitidas en su hogar. El estado federativo que en menor porcentaje señala desaprobación es Sinaloa, con $28.8 \%$; y el que en mayor medida lo hace es Puebla, con $54.9 \%$. Hidalgo reporta $47.1 \%$ de encuestados(as) que señalaron negativamente al cuestionamiento, de forma que ocupa el lugar 11 de 32 en porcentaje de informantes con actitudes discriminatorias.

Con este primer ejercicio, se advierte que es en homosexuales donde recae mayoritariamente el prejuicio en comparación con las mujeres, razón por la cual se ubica en la posición 19 a nivel nacional, a diferencia de la 


\section{Estaría dispuesto o no estaría dispuesto a permitir que en su casa vivieran personas...? Lesbianas}

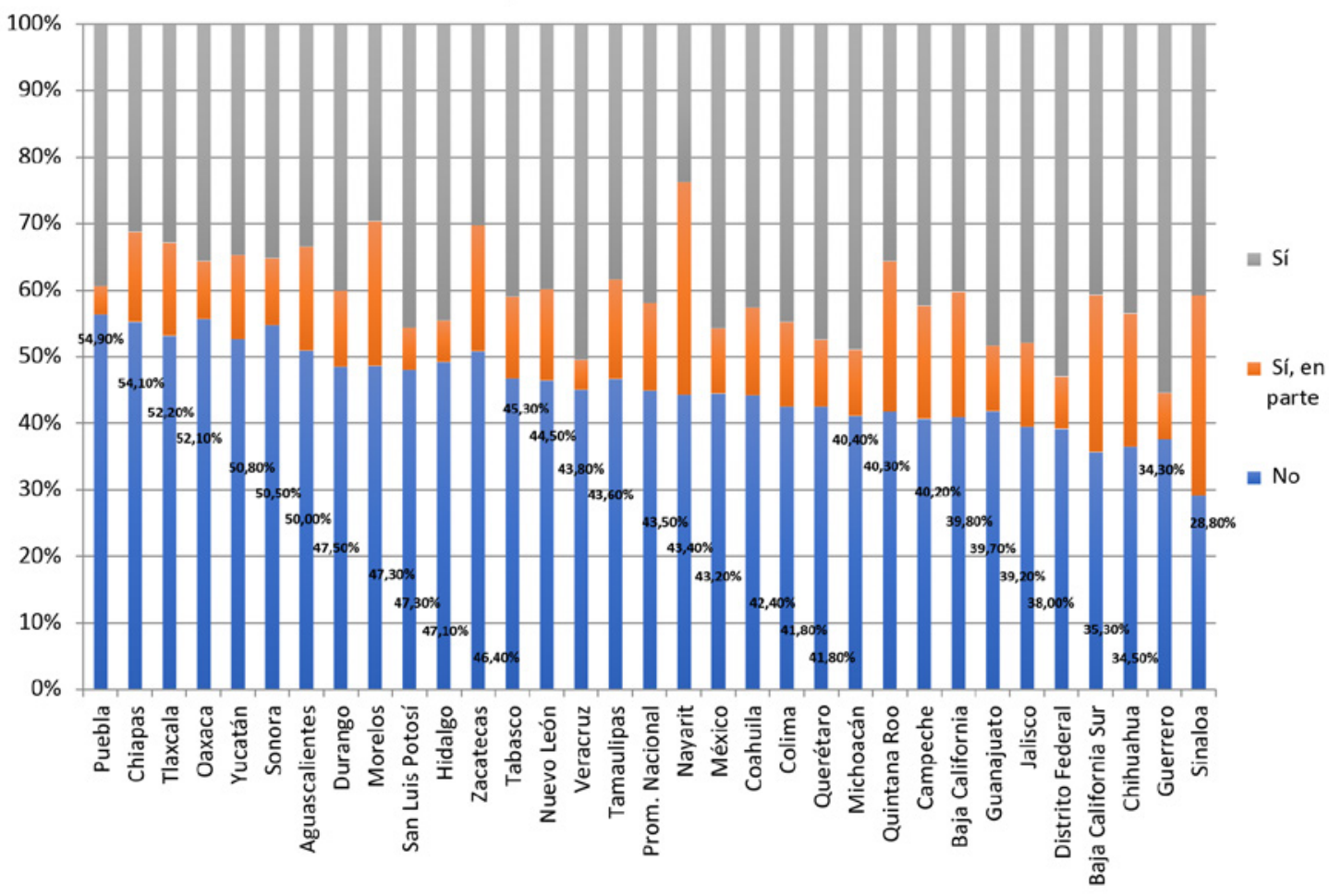

Figura 2. Actitudes discriminatorias contra lesbianas. Comparativo porcentual México y entidades federativas. Fuente: Elaboración propia con base en ENADIS 2010.

12, con estas últimas. Aunado a lo anterior, se observa también que es en estos sectores poblacionales donde recaen mayoritariamente las actitudes discriminatorias, comparativamente al resto de grupos o individuos considerados como susceptibles exclusión (tabla 1). De forma que, tal y como la muestra general reporta, el Estado de Hidalgo, de igual manera, ubica como el principal grupo susceptible de excluir a homosexuales, enseguida lesbianas. 
Tabla

Comparativo de Poblaciones más discriminadas en Hidalgo y México. ENADIS 2010

\begin{tabular}{lcc}
\hline Posición & Hidalgo & México \\
\hline 1 & Homosexuales & Homosexuales \\
& $47.5 \%$ & $44 \%$ \\
2 & Lesbianas & Lesbianas \\
& $47.1 \%$ & $43.5 \%$ \\
3 & Ideas Políticas & VIH-SIDA \\
& $31.4 \%$ & $35 \%$ \\
4 & VIH-SIDA & Extranjeros \\
& $30.9 \%$ & $25.8 \%$ \\
5 & Religión & Ideas políticas \\
& $23.5 \%$ & $25.5 \%$ \\
6 & Extranjeros & Religión \\
& $21.6 \%$ & $24.9 \%$ \\
7 & Cultura distinta & Raza \\
& $19.1 \%$ & $22.8 \%$ \\
8 & Raza & Cultura distinta \\
& $17.5 \%$ & $22.7 \%$ \\
9 & Discapacidad & Discapacidad \\
& $12.3 \%$ & $13.6 \%$ \\
\hline
\end{tabular}

Fuente: Elaboración propia con base en ENADIS 2010.

\section{Construcción de perfiles}

El modelo estadístico proyectó resultados que, conforme a los niveles de confianza a partir de la varianza explicada, permiten aseverar la viabilidad del resultado. La inercia explicada por las interacciones entre variables proyectó valores de .967; haciéndolo válido para el análisis.

Lo mismo para el Alfa de Cronbach, que mide la confiablidad del instrumento, cuyo resultado es de .786, superando los mínimos de consistencia interna (Celina y Campos, 2005). Lo que expone que más del $75 \%$ de las inercias entre los casos son afirmadas (tabla 2). Así, la lectura de los perfiles básicos tiene como principio y referente la dimensión de la variable dependiente en su proximidad con las dimensiones de las variables independientes. 
Tabla 2.

Resumen del modelo

\begin{tabular}{cccc}
\hline \multirow{2}{*}{ Dimensión } & \multirow{2}{*}{ Alfa de Cronbach } & \multicolumn{2}{c}{ Varianza explicada } \\
\cline { 3 - 4 } & & Total (Autovalores) & Inercia \\
\hline 1 & .868 & 3.609 & .602 \\
2 & .652 & 2.191 & .365 \\
Total & & 5.800 & .967 \\
$M$ & $.786^{\text {a }}$ & 2.900 & .483 \\
\hline
\end{tabular}

${ }^{a}$ El Alfa de Cronbach Promedio está basado en los autovalores promedio.

La influencia de cada variable a la construcción del diagrama refirió que la edad, el nivel de instrucción y la religión aportaron más a la definición media en ambas dimensiones. Mientras que el sexo fue la que en menor medida aportó a la construcción del esquema. Por lo que esta última resulta indiferente para la definición de perfiles analíticos, lo cual se corrobora también por el coeficiente de correlación entre esta variable y la dependiente, que es puntualmente nula (-.003).

Es menester ahora referir la distribución de los factores en el diagrama, puntualizando los elementos y su representación para vislumbrar el resultado con desenvoltura. Así que, para el nivel de instrucción, se utilizaron tonos azules; verdes, para la religión; naranjas, para el sexo; gris, para la clase social; amarillo, para la edad; y rojo para la variable dependiente.

La figura 3 permite observar dos perfiles claros de informantes a partir de la referencia de las dimensiones de la variable dependiente. Por la parte negativa del eje $Y$ se ubica el perfil de informante discriminador, mientras que en el segmento positivo se ubica el informante no excluyente. Su composición puede detallarse de la siguiente manera:

1. Perfil discriminante. Clase social de pobreza extrema a clase media. Educación: ninguna, básica y media. Edad: de 36 a 60 años y más. Religión pentecostal: cristiano y católico.

2. Perfil no discriminante. Edad de 18 a 45 años. Escolaridad: más cercano a posgrados y carreras técnicas, aunque también con un 
nivel de educación básica (secundaria). Clase social media baja y de religión evangélica.

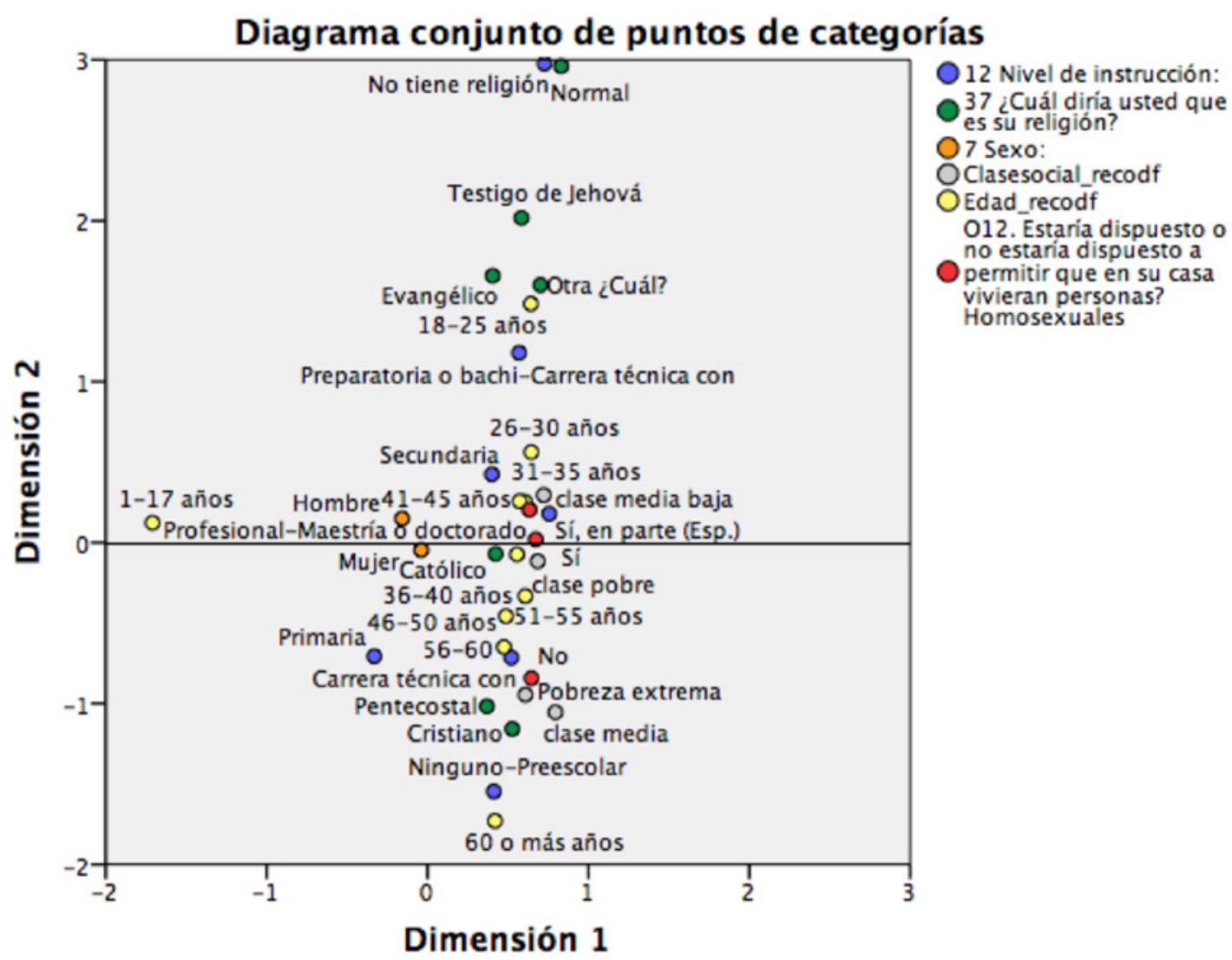

Normalización principal por variable.

Figura 3. Perfiles de informante discriminantes y no discriminantes contra homosexuales.

Fuente: Elaboración propia con base en ENADIS 2010.

Para el caso de la otra variable dependiente, lesbiandad, el resumen del modelo valida igualmente la viabilidad de la técnica, ya que la varianza explicada registró .967 y Alfa de Cronbach es de .786 (tabla 3). 
Tabla

Resumen del modelo

\begin{tabular}{cccc}
\hline \multirow{2}{*}{ Dimensión } & Alfa de Cronbach & \multicolumn{2}{c}{ Varianza explicada } \\
\cline { 3 - 4 } & & Total (Autovalores) & Inercia \\
\hline 1 & .867 & 3.608 & .601 \\
\hline 2 & .652 & 2.191 & .365 \\
\hline Total & & 5.799 & .967 \\
\hline Media & $.786^{\text {a }}$ & 2.900 & .483 \\
\hline
\end{tabular}

${ }^{a}$ El Alfa de Cronbach Promedio está basado en los autovalores promedio.

La influencia de cada factor resulta prácticamente igual al modelo previo, ya que son la edad, el nivel de instrucción y la religión las que en mayor medida condicionan la generación del diagrama en ambas dimensiones. Igualmente, el sexo no es factor determinante por su escasa aportación, ya que el coeficiente de correlación es mínimo (-.024). Así, en la figura 4 se calcula puntualmente la distribución de categorías de la variable, para definir dos perfiles parecidos al modelo previo, en el que se exponen las características del informante discriminador y perfil del agente no excluyente contra este sector.

La distinción, se resume de la siguiente manera:

1. Perfil discriminante. Formación académica sin educación, educación básica (preescolar, primaria y carrera técnica con bachillerato). Religión cristiana, pentecostal y católica. Clase social pobreza extrema, clase pobre y clase media. Edades de 36 a 40 años, 46 a 60 años o más.

2. Perfil no discriminante. Formación académica profesional y posgrados, media superior y secundaria. Religión evangélico, testigo de jehová, otras religiones y sin credos. Clase social media baja. Edad de 18 a 35 y de 41 a 45 años. 


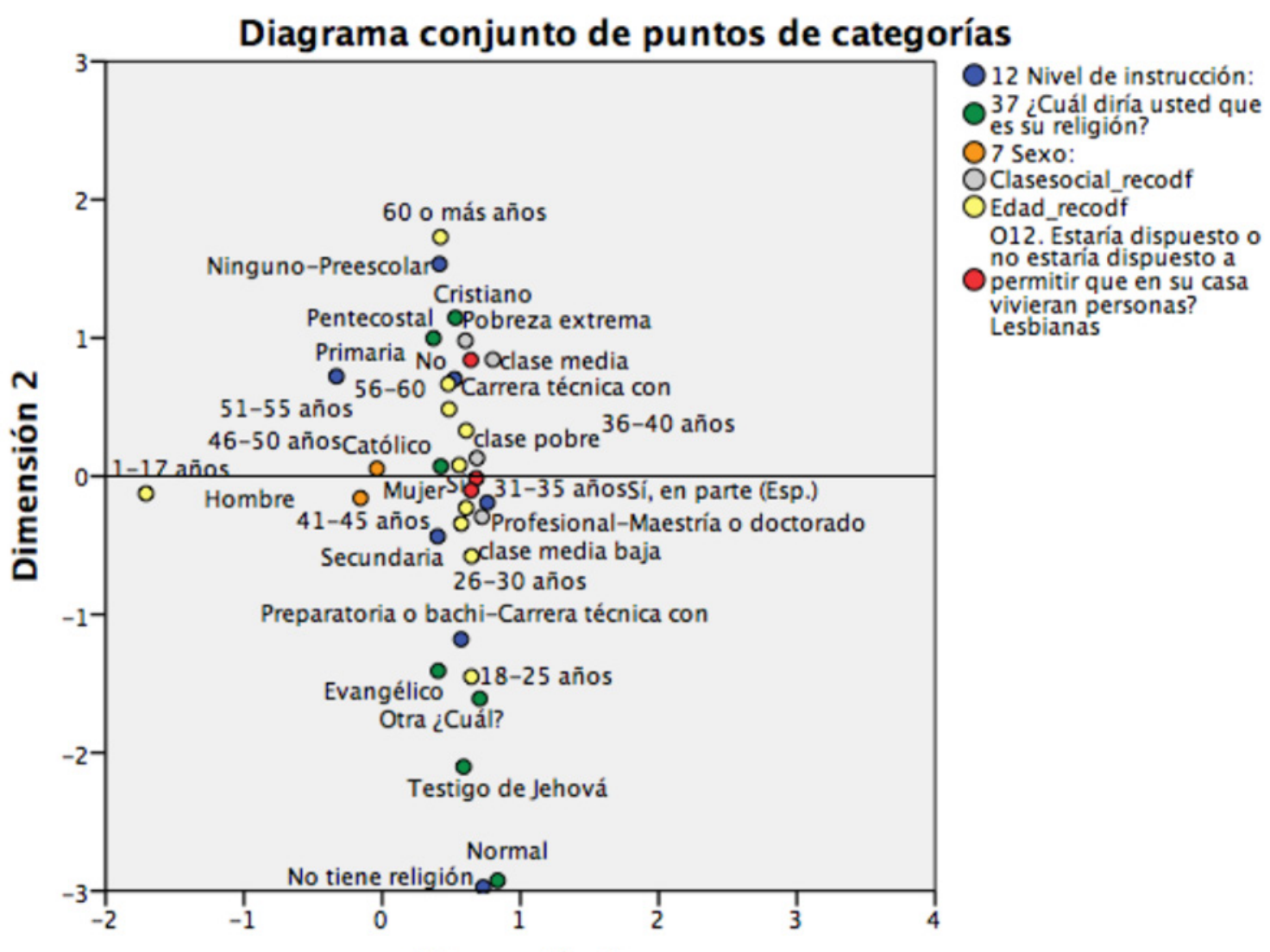

\section{Dimensión 1}

Normalización principal por variable.

Figura 4. Perfiles de informante discriminantes y no discriminantes contra homosexuales.

Fuente: Elaboración propia con base en ENADIS 2010.

\section{DISCUSIÓN}

Comparativa y retrospectivamente a la encuesta de 2005, en el que a nivel nacional el $48 \%$ de informantes refirieron actitudes excluyentes contra homosexuales, en esta edición, el porcentaje es menor en cuatro puntos 
porcentuales. Sin embargo, la constante es que sigue siendo el sector con mayores niveles de prejuicio en contra.

Para la versión de 2010, tras la división de la muestra nacional en dos dimensiones actitudinales contra la homosexualidad y lesbiandad (los que excluyen mucho y poco) en general el promedio de participantes que excluye a homosexuales en el país de $44 \%$; es decir, en cuatro de cada diez. Aunque la entidad que en menor medida lo señala es tres por cada diez, Hidalgo lo hace en casi cico de diez. A pesar de no exceder al máximo (seis de diez), sigue siendo un alto nivel.

De este modo, Hidalgo se ubica en el conjunto de submuestras que más discrimina a estos sectores, ya que para el primer caso se localiza en la posición comparativa 12 de 32, mientras que en lesbianas se ubica en 11 de 32. Sin embargo, es dificultoso concluir que se discrimina más a mujeres no heterosexuales que a varones, porque $47.5 \%$ de informantes que refirieron no aceptar a estos en contraste del $47.1 \%$ con mujeres. Por lo tanto, y a pesar de la poca diferencia, se excluye más a varones que a mujeres en la entidad.

En cuanto a las variables consideradas para la aproximación a los perfiles, se verifica que, si bien el sexo ha sido considerado como factor de control (Gabriel y Banse, 2006; Jarayatne, et al., 2006), para este caso no reporta incidencia considerable para determinar alguna postura. Esto a pesar de que en el perfil de informante que discrimina a homosexuales y lesbianas se ubica a las mujeres de forma marginal; es decir, con una ligera tendencia de mayor intolerancia. Esto, en contrariedad a lo que algunos estudios previos señalan (Fernández y Calderón, 2014; Coppari, et al., 2014; Lozano y Rocha, 2011; LaMar y Kite, 1998).

Con respecto a la edad, se demostró, en ambos perfiles, que prioritariamente es en edades consideradas juveniles y adultos jóvenes donde se localizan los agentes con proximidad a actitudes inclusivas, contribuyendo así a las tesis que refuerza la tendencia. La razón de esto es porque en generaciones más jóvenes se asienta más la tolerancia ante la diversidad sexual que en la adultez o vejez (Piña y Aguayo, 2015; Steffens y Wagner, 2004; Barrientos y Cárdenas, 2010; Coppari, et al., 2014; Acuña-Ruiz y Oyuela, 2006; Herek, 1984); incluso, a sectores de otros tipos de diversidad como migrantes (Semyonov, Raijman, Yom Tov, y Schmidt, 2004). 
La formación escolar es también una variable que corrobora las tendencias empíricas previas, ya que entre mayores niveles de formación escolar, menor es el prejuicio. Tal resultado abona y corresponde también a las propensiones científicas que así lo corroboran (Herek 1984; Herek, 2000; Herek y Capitanio, 1996; Morrison y Morrison, 2011). Según esta concordancia, las personas con mayores niveles educativos conocen una pluralidad de saberes que permiten superar o ampliar las formas elementales inmediatas de vida, haciendo sensible su percepción de las y los demás.

Lo mismo sucedió con las hipótesis concernientes a la clase social, que enuncia la relación positiva entre más alto estrato socioeconómico con la alta aceptación a la diversidad sexual (Pratto, Sidanius, Stallworth, y Malle, 1994; Herek y Capitanio, 1996; Morrison y Morrison, 2011; Swank, Fash, y Frost, 2013). Para estos resultados, es constante la tendencia y proximidad entre estratos altos con actitudes de inclusión. Se ha argumentado que las clases altas tienen una perspectiva cosmopolita que les permite proyectar ventajas de la diversidad a su comprensión, al contrario de las orientaciones parroquiales de las clases bajas (Haubert y Fussel, 2006).

Por último, es evidente la concordancia entre prejuicio y pertenencia a credos religiosos. Si bien en los resultados se verifica la ubicación en el mismo sector de la gráfica a la actitud incluyente y la no adscripción religiosa, tal y como las evidencias empíricas han referido (Piña y Aguayo, 2015; Toro y Varas, 2004; Horvarth y Ryan, 2003); también se localizaron -en ese mismo sector- religiones evangélicas y testigos de Jehová. Mientras que en el conjunto de intolerancia se situaron las religiones pentecostal, cristiana y católica. Esto corresponde con estudios empíricos precedentes que refieren al cristianismo como altamente correlacionado con actitudes discriminatorias hacia homosexuales y lesbianas (Kirpatrick, 1993; Jo-Ann y Wade, 2007); lo mismo en informantes católicos (Jones y Cox, 2011).

En cuanto a las actitudes de las personas de doctrina "testigos de Jehová" se ha demostrado que presentan más propensión a admitir que las personas homosexuales confrontan discriminación como parte de su vida cotidiana, a diferencia de lo que reportan informantes católicos e incluso evangélicos (Jones, Cox y Lienesch, 2017). Por lo tanto, en la dimensión del diagrama en que se ubica el perfil del informante no discriminante, existen tendencias de mayor tolerancia al localizarse evangélicos y testigos de Jehová; pero igualmente se localiza la dimensión de "no tiene 
religión", que se asocia con actitudes de amplia aceptación a gays y lesbianas (Finlay y Walther, 2003). Aunado a lo anterior, es importante referir que, en 2010, el porcentaje de la población de la entidad que profesó la religión católica fue de $87 \%$, haciendo evidente la relación entre creencias cristianas y católicas con obcecaciones contra preferencias o identidades no heteronormativas.

La situación hidalguense con respecto a la discriminación contra homosexuales y lesbianas ha sido calificada por organismos oficiales autónomos como grave (Notimex, 2011). Diversas asociaciones civiles han denunciado públicamente un panorama adverso contra este sector poblacional que carece de garantías concretas para una convivencia pacífica y democrática. El grado de opacidad se refleja a niveles institucionales, ya que del total de 142 leyes existentes en la entidad, solamente 13 -aunque otros estudios aseguran son 19 (Ávila y Serrano, 2017)- son las que refieren, en alguna parte, el término "preferencia sexual", "orientación sexual", identidad de género", "lesbiana", "bisexual", "Gay", "homosexual", "mismo sexo", "transexual", "transgénero" e "intersexual" (Castelán, 2016). Además, el contenido de tales ordenamientos únicamente esboza el acceso a una vida libre de discriminación como ordenanza. Empero, en ninguna de estas se prevén garantías particulares para esta población, lo que confirma la categórica invisibilidad jurídica de las personas de la diversidad sexual (Castelán, 2016).

Es importante señalar, también, que paradójicamente a lo referido en los datos de la entidad, el organismo local encargado de atender denuncias únicamente reporta quince quejas por discriminación contra personas de la comunidad LGBTTTI entre 2010 y 2017 (Chávez, 2017). Lo anterior discrepa con lo que señala una muestra estadística de personas en esta comunidad, levantada en la ciudad capital de la entidad (Pachuca de Soto) en 2016, la cual reporta que una de cada dos persona ha sido discriminada en espacios educativos; el $70.5 \%$ relató recibir insultos y el $15 \%$ burlas; el $80 \%$ ha sido violentado física y verbalmente en la calle por personas desconocidas y el $12.5 \%$ por algún familiar.

En cuanto al espacio laboral, una de cada dos personas no accede a prestaciones sociales; al $77 \%$ de los varones homosexuales se les solicitó pruebas de $\mathrm{VIH}$ en las entrevistas para conseguir empleo, el $42 \%$ han sido amenazados con ser despedidos a causa de su orientación sexual 
y el $13.9 \%$ reportó haber sido detenido por policías solo por su condición (Castelán, 2017). Ante tales datos, aparentemente la invisibilidad así como represión es cotidiana pero no es reflejada en los informes oficiales.

Estudios empíricos clásicos (Herek, 1984; Herek, 2000; Steffens y Wagner, 2004; LaMar y Kite, 1998) han señalado la relación directa entre la apertura, contacto, visibilidad, así como convivencia entre personas heterosexuales y no heterosexuales para incentivar actitudes incluyentes y de respeto. Si persiste el prejuicio en un contexto donde las instancias estatales omiten intervenir para paliar el fenómeno discriminatorio, la sistemática estigmatización acarrea consecuencias de salud pública porque provoca suicidio (Clements-Nolle, Marx y Katz, 2006); mayor desigualdad social, porque son menos contratados en empleos formales (Hebl, Foster, Mannix, y Dovidio, 2002; Tilcsik, 2011) y con inferiores salarios (Lee, 1995; Berg y Lien, 2002; Castelán, 2017). Lo que se traduciría en serios problemas que perpetuarían los altos índices de pobreza, marginalidad y desigualdad.

Esto supone que la injusticia cultural está estrechamente relacionada con injusticia distributiva y se debe al aún no reconocimiento de la diversidad identitaria en los marcos simbólicos vigentes que socavan las posibilidades de un estado diverso, incluyente y equitativo. De modo que ambas injusticias deben ser consideradas en las políticas públicas orientadas a este sector. Sin embargo, en México se concentra la atención en el reconocimiento sin distribución de riqueza, lo que omite necesidades específicas que merman posibilidades reales de combate a la desigualdad (Galindo, 2017) así como de ciudadanía sustantiva, ya que no se les admite como agentes iguales, válidos y con capacidades morales como el resto (Honneth, 1997).

\section{CONCLUSIONES}

El objetivo de este trabajo es identificar los niveles actitudinales discriminatorios de informantes del estado de Hidalgo con respecto a la homosexualidad y lesbiandad, contrastándolos con el resto de entidades del país. En segundo lugar, se propuso construir un perfil analítico de informante con tendencias excluyentes. 
Hidalgo se posiciona entre las entidades que más discrimina a homosexuales y lesbianas, según la Encuesta Nacional sobre Discriminación 2010. Pero también se corrobora, a partir de las denuncias, que fuentes oficiales registraron entre 2010 y 2017 ya que en su totalidad se concentran en ataques hacia esta población. Esto, aunado a la invisibilidad en las instituciones jurídicas que no se contempla su existencia en la mayoría de ordenamientos. El perfil de informante excluyente es de una persona adulta -entre 36 años de edad y más-, de formación académica que va de nula a básica, varón, de clase social en pobreza extrema a media y de creencias cristianas, católicas y/o pentecostal.

El aporte de este trabajo pretende coadyuvar a la generación de conocimiento científico social de la situación de discriminación en una de las entidades mexicanas que pueda ser útil para la comprensión del fenómeno de forma un poco más detallada a los estrictos resultados descriptivos que la encuesta proporciona. Con esto se contribuye a la visibilidad de los grupos estructuralmente discriminados y así se nutren las condiciones elementales del reconocimiento con fines de conseguir ciudadanía.

\section{AGRADECIMIENTOS}

Esta indagación es resultado del proyecto de Investigación "Diagnóstico de la discriminación en Pachuca de Soto, Hidalgo y la zona conurbada" financiado por el Programa para el Desarrollo Profesional Docente de la Secretaría de Educación Pública de México con número de registro en la Universidad Autónoma del Estado de Hidalgo: UAEH-EXB-148.

\section{REFERENCIAS}

Acuña-Ruiz, a. y Oyuela, R. (2006). Diferencias en los prejuicios frente a la homosexualidad masculina en tres rangos de edad en una muestra de hombres y mujeres heterosexuales. Psicología desde el Caribe, (18), 58-88. Recuperado de http://www.redalyc.org/articulo. oa? id=21301804

Aguayo, J. (14 de mayo de 2014). Las clases sociales en México según la secretaría de economía... ¿Usted a cuál pertenece?. Tribuna. Periodismo libre y objetivo. Recuperado de http://www.jorgevargas.com. mx/archivo/20140514/8635.htm 
Alexandre, A. (2001). ¿Qué dice la biblia sobre la homosexualidad? Teología y Vida, 42(4), 277-398. doi: 10.4067/S0049-34492001000400001

Ávila, A. y Serrano, L. (junio de 2017). Situación actual de la diversidad sexual en el estado de Hidalgo. En Academia Hidalguense de Educación y Derechos Humanos A.C. Primer Festival Estatal por la Diversidad Sexual. Pachuca de Soto, Hidalgo. México.

Barba, C. (2012). Encuesta Nacional sobre Discriminación 2010. Espiral, 19(54), 261-270. Recuperado de http://www.scielo.org.mx/scielo. php?script=sci_arttext\&pid=S1665-05652012000200011

Barrientos, J. (2016). Situación social y legal de gays, lesbianas y personas transgénero y la discriminación contra estas poblaciones en América Latina. Sexualidad, Salud y Sociedad-Revista Latinoamericana, (22), 331-354. doi: 10.1590/1984-6487.sess.2016.22.15.a

Barrientos, J. y Cárdenas, J. (2010). Aceptación y validación de la escala Likert de actitudes de heterosexuales hacia homosexuales (HATH) en una muestra de estudiantes universitarios chilenos. Sexualidad, Salud y Sociedad-Revista Latinoamericana, (5), 30-49. Recuperado de http://www.redalyc.org/articulo.oa?id=293323015003

Becerro-Acosta, J. (20 de mayo de 2016). Reportan mil 310 homicidios por homofobia en 20 años. Milenio Diario. Recuperado de http://www.milenio.com/politica/homofobia_homicidios-Discriminacion_social-crimenes_de_odio-odio_homofobico-LGTB_0_740925943.html

Berg, N. y Lien, D. (2002). Measuring the effect of sexual orientation on income: evidence of discrimination. Contemporany Economic Policy, 20(4), 394-414. Recuperado de http://www.otago.ac.nz/economics/ otago113959.pdf

Cabral, B. (2017). Sexo, poder y género. Un juego con las cartas marcadas. México: CAPUB-Fundación Editorial el Perro y la Rana- Centro Educativo de Calidad para la Integración Humana.

Castelán, R. (2016). Investigación para la realización de un diagnóstico. Atención a personas lesbianas, gays, bisexuales y trans en México. Informe del estado de Hidalgo. México: Fundación Arcoiris. 
Recuperado de http://seiinac.org.mx/wp-content/uploads/2016/11/ Informe_LGBTTTI_Hidalgo.pdf

Castelán, R. (2017). Atención a personas LGBT en Hidalgo. En R. Zapata (Presidencia). XIII Congreso Nacional sobre Empoderamiento Femenino. Pachuca de Soto, Hidalgo. México. Recuperado de https:// www.uaeh.edu.mx/xiii_congreso_empoderamiento_fem/documentos/pdf/C058.pdf

Celina, H. y Campos, A. (2005). Aproximación al uso del coeficiente de alfa de Cronbach. Revista Colombiana de Psiquiatria, 34(4), 572580. Recuperado de http://www.redalyc.org/pdf/806/80634409.pdf

Chávez, A. (18 de mayo de 2017). Reportan15 quejas de discriminación a comunidad LGBTTI. La Silla Rota. Recuperado de https://lasillarota.com/estados/ reportan-15-quejas-de-discriminacion-a-comunidad-lgbtti/151428

Clements-Nolle, K., Marx, R. y Katz, M. (2006). Attempted suicide amog transgender persons. Journal of Homosexuality, 51(3), 53-69. doi: 10.1300/J082v51n03_04

Colino, C. (2009). Método comparativo. En Reyes, R. (Dir.). Diccionario crítico de Ciencias Sociales. Terminología científico-social, Tomo 1. México/Madrid: Plaza y Valdés. Recuperado de http://pendientedemigracion.ucm.es/info/eurotheo/diccionario/M/metodoc omparativo_a.htm

Consejo Nacional para Prevenir la Discriminación y Secretaría de Desarrollo Social. (2005). Primera Encuesta sobre Discriminación en México. Recuperado de http://www.conapred.org.mx/userfiles/files/Presentacion_de_la_Encuesta_final.pdf

Consejo Nacional para Prevenir la Discriminación. (2011). Encuesta Nacional sobre discriminación en México. Enadis 2010. Resultados generales. México: Consejo Nacional para prevenir la Discriminación. Recuperado de https://www.conapred.org.mx/documentos_cedoc/ Enadis-2010-RG-Accss-002.pdf 
Constitución Política de los Estados Unidos Mexicanos. (2016). México: Diario Oficial de la Federación. Recuperado de http://www.ordenjuridico.gob.mx/Constitucion/cn16.pdf

Coppari, N., Arcondo, G., Bagnoli, L., Chavez, M., Corvalán, M., Enciso, A., Melgarejo, K. y Rodríguez, X. (2014). Prejuicio y distancia social hacia la homosexualidad en universitarios de psicología de Paraguay. Salud \& Sociedad, 5(3), 240-252. Recuperado de http://www. redalyc. org/articulo.oa?id $=439742476001$

Diario Oficial de la Federación [DOF]. (2001). Constitución Política de los Estados Unidos Mexicanos de 1917 (última Reforma DOF 14/08/2001). Recuperado de http://www.dof.gob.mx/nota_detalle. php?codigo $=762221 \&$ fecha $=14 / 08 / 2001$

Durkheim, E. (2000). Las formas elementales de la vida religiosa. México: Colofón.

Fernández, M. y Calderón, J. (2014). Prejuicio y distancia social hacia personas homosexuales por parte de jóvenes universitarios. Revista Puertorriqueña de Psicología, 25(1), 52-60. Recuperado de http:// www.redalyc. org/articulo.oa?id=233237909004

Fernández, P. (2014). Dinámica demográfica 1990-2010 y proyecciones de población 2010-2030. Hidalgo. México: Consejo Nacional de Población. Recuperado de http://www.conapo.gob.mx/work/models/ CONAPO/Proyecciones/Cuadernos/13_Cuadernillo_Hidalgo.pdf

Finlay, B. y Walther, C. (2003). The relation of religious affiliation, service attendance, and other factor homophobic attitudes among university students. Review of Religious Research, 44(4), 370-393. doi: $10.2307 / 3512216$

Franco, L. M., (2012). La migración en el estado de Hidalgo. Un enfoque de desarrollo regional. Pachuca de Soto: Universidad Autónoma del Estado de Hidalgo.

Gabriel, U. y Banse, R. (2006). Helping behavior as a subtle measure of discrimination against lesbians and gay men: German data and a 
comparison across countries. Journal of Applied Psychology. 36(3), 690-707. doi: 10.1111/j.0021-9029.2006.00025.x

Galindo, L. (2017). Reflexiones sobre políticas públicas: diversidad sexual en México. El Cotidiano, (202), 73-83. Recuperado de https://www. redalyc.org/html/325/32550024007/

Goffman, E. (2006). Estigma. La identidad deteriorada. Buenos Aires, Argentina: Amorrortu.

González, J. (25 de enero de 2015). Salarios, clases sociales e informalidad. Vanguardia. Recuperado de https://www.vanguardia.com.mx/ columnas-salariosclasessocialeseinformalidad-2257012.html

Greenacre, M. y Hastie, T. (1987). The geometric interpretation of correspondence análysis. Journal of the American Statistical Association, 82(398), 437-447. doi: 10.2307/2289445

Haubert, J. y Fussell, E. (2006). Explaining pro-immigrant sentiment in the U.S.: Social class, cosmopolitanism, and percepción of immigrants. International Migration Review, 40(3), 489-507. doi: 10.1111/j.1747-7379.2006.00033.x

Hebl, M., Foster, J., Mannix, L., y Dovidio, J. (2002). Formal and interpersonal discrimination: a field study of bias toward homosexual applicants. Personality and Social Psychology Bulletin. 28(6), 815-825. doi: $10.1177 / 0146167202289010$

Herek, G. (1984). Beyond "homophobia". Journal of Homosexuality, 10(12), 1-21. doi: 10.1300/J082v10n01_01

Herek, G. (2000). The psychology of sexual prejudice. Current Directions in Psychological Science, 9(1), 19-22. doi: 10.1111/1467-8721.00051

Herek, G. (2004). Beyond "Homophobia": Thinking about sexual prejudice and stigma in the twenty-first century. Sexuality Research \& Social Policy, 1(2), 6-24. doi: 10.1525/srsp.2004.1.2.6

Herek, G. y Capitanio, J. (1996). "Some of my best friends" Intergroup contact, conceleable stigma, and heterosexuals' attitudes oward gay 
men and lesbians. Personality and Social Psichology Bulletin, 22(4), 412-424. doi: 10.1177/0146167296224007

Herek, G. M., Cogan, J. C., Gillis, J. R., y Glunt, E. K. (1998). Correlates of internalized homophobia in a community sample of lesbians and gay men. Journal of the Gay \& Lesbian Medical Assn, 2(1), 17-25. Recuperado de http://psychology.ucdavis.edu/rainbow/html/JGLMA_1998_pre.pdf

Herek, G. M., Gillis, J. R., y Cogan, J. C. (2015). Internalized stigma among sexual minority adults: Insights from a social psychological perspective. Stigma and Health, 1(S), 18-34. Recuperado de http://dx.doi. org/10.1037/2376-6972.1.S.18

Honneth, A. (1997). La lucha por el reconocimiento. Por una gramática moral de los conflictos sociales. Barcelona, España: Crítica.

Horvarth, M. y Ryan, A. (2003). Antecedents and potential moderartors of the relationship between attitudes and hiring discrimination on the basis of sexual orientation. Sex Roles, 48(3-4), 115-130. doi: 10.1023/A:1022499121222

Inglehart, R. y Welzel, C. (2006). Modernización, cambio cultural y democracia. La secuencia del desarrollo humano. Madrid, España: CIS.

Jayaratne, T., Ybarra, O., Sheldon, J., Brown, T., Feldbaum, M., Pfeffer, C., y Petty, E. (2006). White americans' genetic lay theories of race differences and sexual orientation: Their relationship with prejudice toward blacks, and gay men and lesbians. Group Process Intergroup Relat, 9(1), 77-94. doi: 10.1177/1368430206059863

Jo-Ann, T. y Wade, R. (2007). The Relationship Between Religious Orientation, Right-Wing Authoritarianism, and Implicit Sexual Prejudice. The International Journal for the Psychology of Religion, 17(2), 99-120. Recuperado de http://www.baylorisr.org/wp-content/uploads/tsang_ relationship.pdf

Jones, M. y Sullivan, G. (2008). Psychiatric disorder or straight prejudice. The role of education in overcoming homophobia. Journal of Gay \& Lesbian Social Services, 14(2), 95-105. doi: 10.1300/J041v14n02_08 
Jones, R. y Cox, D. (2011). Catholic attitudes on gay and lesbian issues: A comprehensive portrait from recent research. Washintong, DC: Public Religion Research Institute. Recuperado de http://friendsandfamilyplan.org/wp-content/uploads/2012/10/Public-Religion-Catholics-and-LGBT-Issues-Survey-Report.pdf

Jones, R., Cox, D., y Lienesch, R. (2017). Who Sees Discrimination? Attitudes on Sexual Orientation, Gender Identity, Race, and Immigration Status / Findings from PRRI's American Values Atlas. PRRI. 2017. Recuperado de https://www.prri.org/research/americans-views-discrimination-immigrants-blacks-lgbt-sex-marriage-immigration-reform/

Kinsey, A., Pomeroy, W., y Martin, C. (2003). Sexual Behavior in the human male. American Journal of Public Healt, 6(93), 894-898. Recuperado de http://ajph.aphapublications.org/doi/full/10.2105/AJPH.93.6.894

Kirpatrick, L. (1993). Christian orthodoxy and intrinsic religious orientation as a predictor of a discriminatory attitude. Journal for the Scientific Study of Religion. 32(3), 256-268. doi: 10.2307/1386664

LaMar, L. y Kite, M. (1998). Sex differences in attitudes toward gay men and lesbians: A multidimensional perspective. The Journal of Sex Research, 35(2), 189-196. doi: 10.1080/00224499809551932

Laythe, B., Finkel, D. y Kirckpatrick, L. (2001). Predicting prejudice from religious fundamentalism and right-wing autoritarianism: A multiple-regression approach. Journal for the Scientific Study of Religion, 40(1), 1-10. Recuperado de http://www.jstor.org/stable/1388176

Lee, M. (1995). The Wage Effects of Sexual Orientation Discrimination. Industrial and Labor Relations Review, 48(4), 726-739. doi: $10.2307 / 2524353$

Lijphart, A. (1971). Comparative politics and the comparative method. American Political Science Review, 65(3), 682-693. doi: 10.2307/1955513

López, P. (1996). La construcción de tipologías: metodología de análisis. Papers. Revista de Sociología, (48), 9-29. Recuperado de https:// ddd.uab.cat/pub/papers/02102862n48/02102862n48p9.pdf 
Lozano, I. y Rocha, T. (2011). La homofobia y su relación con la masculinidad hegemónica en México. Revista Puertorriqueña de Psicología, 22, 101-121. Recuperado de http://www.redalyc.org/articulo. oa? $\mathrm{id}=233222354002$

Lozares, C. (1990). La tipología en sociología, más allá de la simple taxonomía: conceptualización y cálculo. Papers. Revista de Sociología, (34), 139-163. Recuperado de https://ddd.uab.cat/pub/papers/02102862n34/02102862n34p139.pdf

Mills, M., Van de Bunt, G., y De Brujin, J. (2006). Comparative research. Persistent problems and promising solutions. International Sociology, 21(5), 619-631. doi: 10.1177/0268580906067833

Mogrovejo, N. (2000). Un amor que se atrevió a decir su nombre. La lucha de las lesbianas y su relación con los movimientos homosexual y feminista en América Latina. México: Plaza y Valdés/CDAHL.

Monsiváis, C. (2012). La homofobia y sus prejuicios. En J. Muñoz (Coord.). Homofobia. Laberinto de la ignorancia (pp. 23-31). México: UNAM-Centro de Investigaciones Interdisciplinares en Ciencias y Humanidades-Colegio de Ciencias y Humanidades.

Morrison, M. y Morrison, T. (2011). Sexual orientation bias toward gay men and lesbian women: Modern homonegative attitudes and their association with discriminatory behavioral intentions. Journal of Applied Social Psychology, 41(11), 2573-2599. doi: 10.1111/j.1559-1816.2011.00838.x

Notimex. (12 de Julio de 2011). Presentan encuesta sobre discriminación en Hidalgo. SDP Noticias. Recuperado de http://www.sdpnoticias.com/notas/2011/07/12/ presentan-encuesta-sobre-discriminacion-en-hidalgo

Pantoja,S.(11demayode2015). México, segundolugarencrímenesporhomofobia. Proceso. Recuperado de http://www. proceso.com.mx/403935/ mexico-segundo-lugar-mundial-en-crimenes-por-homofobia 
Piña, J. y Aguayo, H. (2015). Homofobia entre estudiantes universitarios de México. Región y Sociedad, 27(64), 5-35. Recuperado de http:// www.redalyc.org/pdf/102/10241350001.pdf

Pratto, F., Sidanius, J., Stallworth, L., y Malle, B. (1994). Social dominance orientation: A personality variable predicting social and political attitudes. Journal of Personality and Social Psychology, 67(4), 741-763. doi: 10.1037/0022-3514.67.4.741

Rivera, E., Tirado, G., y Olea, P. (2014). La memoria femenina desde la disidencia sexual. Cuadernos de Inter.c.a.mbio sobre Centroamérica y el Caribe, 11(2), 47-65. Recuperado de http://www.redalyc.org/ pdf/4769/476947242003.pdf

Rubin, G. (2003). El tráfico de mujeres. Notas sobre la economía política del sexo. En M. Lamas (Comp.). El género. La construcción cultural de la diferencia sexual. (pp. 35-96). México: Miguel Ángel Porrúa y Universidad Nacional Autónoma de México.

Semyonov, M., Raijman, R., Yom Tov, A., y Schmidt, P. (2004). Population size percived threat, and exclusión: a multiple-indicators analysis of attitudes toward foreingers in Germany. Social Science Research, 33(4). 681-701. doi: 10.1016/j.ssresearch.2003.11.003

Serret, E. (2001). El género y lo simbólico. La constitución imaginaria de la identidad femenina. México: Universidad Autónoma Metropolitana - Azcapotzalco.

Serret, E. (2004). Mujeres y hombres en el imaginario social. La impronta de género en las identidades. En M. E. Cosio (Coord.). Mujeres y sociedad en el México contemporáneo. Nombrar lo innombrable. (pp. 43-70). México: Miguel Ángel Porrúa, Tec. De Monterrey y Cámara de Diputados LIX Legislatura.

Smith-Castro, V. y Molina-Delgado, M. (2011). Actitudes hacia el matrimonio y la unión civil gay en Costa Rica ¿Religiosidad, homofobia, autoritarismo o desconocimiento? Interamerican Journal of Psychology, 45(2), 133-143. Recuperado de https://journal.sipsych.org/index. php/IJP/article/view/143/pdf 
Sourial, N., Wolfson, C., Zhu, B., Quail, J., Karunananthan, S., Bandeen-Roche, K., y Bergman, H. (2010). Correspondence analysis is a useful tool to uncover relationship among categorical variables. Journal of Clinical Epidemiology, 63(6), 638-646. doi: 10.1016/j. jclinepi.2009.08.008

Steffens, M. y Wagner, C. (2004). Attitudes toward lesbians, gay men, bisexual women, and bisexual men in Germany. The Journal of Sex Research, 41(2), 137-149. Recuperado de http://www.jstor.org/ stable/3813648

Swank, E., Fash, B., y Frost, D. (2013). Region, social identities, and disclosure practices as a predictors of heterosexist discriminations against sex minorities in the United States. Sociological Inquiry, 83(2), 238258. doi: 10.1111/soin. 12004

Tena, O. (2012). Análisis ético de la homofobia. En J. Muñoz (Coord.). Homofobia. Laberinto de la ignorancia (pp. 91-107). México: UNAM-Centro de Investigaciones Interdisciplinares en Ciencias y Humanidades-Colegio de Ciencias y Humanidades.

Tilcsik, A. (2011). Pride and prejudice: Employment discrimination against openly gay men in the United States. American Journal of Sociology, 117(2), 586-626. Recuperado de http://www.jstor.org/ stable/10.1086/661653

Toro, J. y Varas, N (2004). Los otros: prejuicio y distancia social contra hombres gay y lesbianas en una muestra de estudiantes de nivel universitario. International Journal of Clinical and Health Psychology, 4(3), 537-551. Recuperado de http://www.redalyc.org/articulo. oa? id=33740306

Valencia, J. y Romero, R. (2017). Las lesbianas en México continúan invisibilizadas en las políticas públicas. El Cotidiano, (202), 85-94. Recuperado de http://www.redalyc.org/html/325/32550024008/ 
Vargas, P. (2007). Hidalgo. Agenda de derechos humanos. Haciala formación de una política pública local. Pachuca de Soto: Academia Hidalguense de Educación y Derechos Humanos. Recuperado de https://issuu. com/albertobuitre/docs/libro._derechos_humanos_en_hidalgo_2007

Vigour, C. (2011). Comparision. A fundational approach in the social sciences. En Queúdraogo, J. y Cardoso, C. (Eds.). Readings in methodology. African perspectives. (p. 215-246). Senegal: Council for the development of social sciences in África. Recuperado de http://www. codesria.org/spip.php?article1502\&lang $=f r$

Visauta, V. y Martori, J. (2003). Análisis estadístico con SPSS para Windows. Vol. II. Estadística multivariante. Madrid: McGraw Hill. 
